\title{
Gains and limitations of a connected tracking solution in the perioperative follow-up of colorectal surgery patients
}

\author{
F. Agri, D. Hahnloser, N. Demartines and M. Hübner \\ Department of Visceral Surgery, Lausanne University Hospital (CHUV), University of Lausanne (UNIL), Lausanne, Switzerland \\ Received 22 July 2019; accepted 25 January 2020; Accepted Article online I February 2020
}

\begin{abstract}
Aim The means to target shorter hospital stay include information technology strategies to improve communication between caregivers and patients in order to limit potentially avoidable readmissions. The aim of the present study was to analyse the benefits and limitations of a smartphone-based connected tracking solution in the perioperative follow-up of colorectal surgery patients.
\end{abstract}

Method This was a retrospective monocentric cohort study of consecutive patients after colorectal surgery between February and December 2018. The mobile health application included information delivery and daily structured questionnaires on a personalized patient electronic profile, before the hospital stay and for 7 days post-discharge. The medical team answered automatic alerts in real time.

Results A total of 93 eligible patients were approached and 36 had to be excluded (26 no smartphone, five no email, five not French speaking). Among the potential users, $50(88 \%)$ engaged in an mHealth app and seven refused. Of these 50 patients, seven dropped out. Of the remaining 43 patients, the app detected 12 adverse events, and $10(83 \%)$ were handled through the app.
Healthcare providers responded to patient-generated alerts after a median time of 90 min (range 9$448 \mathrm{~min}$ ). Patients' mean satisfaction level was $4 \pm 0.97$ out of 5 .

Conclusion In total, $88 \%$ of smartphone-equipped patients showed a willingness to engage in mHealth. Reasons for exclusion were the absence of connection tools and a language barrier. Patients who responded to the survey were satisfied with the solution and $83 \%$ of post-discharge adverse events were solved through the app, avoiding emergency consultations.

Keywords adverse events, colorectal surgery, complications, connected follow-up, information technology, mHealth app, perioperative follow-up

What does this paper add to the existing literature?

This work contributes to enriching the literature on the most current topic: mHealth. It reports on the experience of using an mHealth app in clinical practice and highlights the gains and limitations of new information technology strategies in the field of colorectal surgery.

\section{Background}

After two decades of telemedicine and the release of the first medical app in $2010[1,2]$, the production of health-related connected solutions has increased dramatically. As stated by the World Health Organization, the term mHealth refers to a 'medical and public health practice, supported by mobile devices such as mobile phones, patient monitoring devices, personal digital assistants, and other wireless devices' [3]. Among the 165000 (2018) available 'medical' apps,

Correspondence to: Professor Nicolas Demartines, Department of Visceral Surgery, Lausanne University Hospital CHUV, Bugnon 46, I0II Lausanne, Switzerland.

E-mail: demartines@chuv.ch only $2 \%$ have the feature to connect the consumer to a healthcare provider and most of them are dedicated to the management of chronic diseases $[4,5]$. These tools are increasingly seen as a solution for prevention and early recognition of post-discharge complications, whilst at the same time potentially reducing the length of stay in hospital and avoidable readmissions. In fact, a shortened length of stay due to enhanced recovery after surgery (ERAS) pathways raises the question of monitoring early post-surgical complications [6]. Furthermore, over $80 \%$ of readmissions are actually associated with new post-discharge complications $[7,8]$, and it is recognized that the early treatment of complications leads to better outcomes [9- 
11]. This is of importance, because the length of stay is gradually reduced due to medical innovations and economic constraints $[12,13]$.

Recently, in selected patients, Gignoux et al. demonstrated the feasibility of outpatient colectomy with a combination of an enhanced recovery protocol and home monitoring, including a medical app and a daily nurse visit $[14,15]$. Further recent studies demonstrated the feasibility of using a mobile app for the monitoring of surgical patients, focusing on outpatients [16-19] and low complexity procedures [20].

The aim of the present work was to study the feasibility, potential benefits and limitations of mHealth for connected follow-up in patients after colorectal surgery.

\section{Method}

This was a retrospective monocentric cohort study of consecutive patients after colorectal surgery between February and December 2018. The approached patients were offered the opportunity to use Maela ${ }^{\circledR}$ (Lyon, France) a connected tracking app. Each user provided informed consent prior to using the mHealth app. The study was carried out retrospectively, at the end of the pilot, with approval from the institutional review board to re-access the patient data (CER-VD \# 2019-00365, Ethics Committee Vaud, Switzerland).

Adult patients undergoing elective colorectal surgery including segmental and total colectomies, rectal resections and stoma procedures were eligible. Further requirements were the possession of a smartphone and an email address. Patients not understanding French were excluded.

Maela ${ }^{\circledR}$ is provided by a French company and uses the storage capacity of Santeos ${ }^{\circledR}$ (Bezons, France), an ISO 9001, 20000 and 27001 certified company. The connected follow-up offered continuous ERAS information delivery to the patient, as well as daily structured questionnaires. Depending on the patient's responses, alerts were generated and sent by email, short messages (SMS) or directly to the healthcare provider app, according to the predefined wishes of the user. ERAS information was available to patients on their personal electronic profile (e-profile) and was accessible by smartphone or internet. For the purpose of the present pilot study, three structured protocols were created. Each protocol allowed active search for specific complications, such as pain, venous thrombo-embolic event, surgical site infection (SSI), bowel obstruction or ostomy problems. The first protocol involved colorectal resections. Included operations were elective right and left colectomy, transverse colectomy, total colectomy, proctocolectomy, sigmoidectomy and low anterior resection by both laparoscopy or laparotomy. The second protocol involved ostomy procedures. Included operations were those of the first protocol with the addition of an ileostomy or colostomy. The third protocol involved stoma closures (ileostomy or colostomy). Questionnaires were available on patients' smartphones starting 2 days prior to hospital admission until 7 days post-discharge. Reminders were sent as SMS. Prior to hospital admission, patients received structured pre-admission information. During the hospital stay, they had access to the ERAS electronic brochure. Post-discharge questionnaires were automatically available to patients after discharge on their e-profile each day for seven consecutive days. All items were closed questions with predefined answers. A 1-10 pain scale was used to assess the pain. In addition, it was possible to transmit body temperature measurements and photos of the wound. Patients were also able to use built-in messaging to report any adverse event ( $\mathrm{AE}$ ) occurring between the completion of questionnaires. An $\mathrm{AE}$ was defined as any deviation of the predefined 'normal' pathway. For every $\mathrm{AE}$, either an automated response was created or an alert was sent to the healthcare providers. Alerts were generated when the patient's answer deviated from a predefined 'normal' post-discharge evolution (i.e. body temperature $>38^{\circ} \mathrm{C}$, pain $\geq 6 / 10$ or vomiting). Moreover, in cases of wound problems, the algorithm asked the patient to upload a photo on their e-profile. Due to inherent pilot limitations, answers and alerts were monitored during office hours only $(8$ am $-5 \mathrm{pm}$, Monday to Friday). For this reason, outside of these hours and during the weekend, patients followed the standard procedure and called the surgical ward directly.

A team of two colorectal consultants, two clinical nurses and one specialized ERAS nurse received formal training to manage patient-generated alerts by smartphone or desktop computer. A third senior surgeon acted as a local app administrator. The colorectal surgeons enrolled eligible patients during outpatient consultations and reasons for exclusion or refusal were documented. The recruitment period was between February and December 2018. The study ended in February 2019, when all included patients had passed their post-surgical visit, on average 30 days after the primary hospital stay. It should be emphasized that the institutional postoperative follow-up was not modified. All patients were seen as outpatients for postoperative control, 1 month after discharge. In addition, patients without a general practitioner were seen systematically 15 days post-discharge.

Variables were extracted from the hospital's electronic patient record and included age at admission, gender, surgical intervention, the malignant or benign 
nature of the diagnosis, date of entrance, date of operation, surgical intervention and date of discharge, as well as the 30-day post-discharge complications. Variables collected from the electronic platform of the connected solution included duration of the connected follow-up, response rate to the daily questionnaires, delay between patient-generated alerts and physician assistant's (PA's) response, number of messages exchanged through the platform and number of complications detected by the connected follow-up. A satisfaction questionnaire was also proposed on the patient's e-profile at the end of the follow-up. Possible answers were not satisfied $=1$, little satisfied $=2$, somewhat satisfied $=3$, satisfied $=4$ and very satisfied $=5$.

Statistical analyses and graphics were performed using Microsoft Office Excel's 2007 descriptive statistic tool.

\section{Results}

From February to December 2018, retrospective screening identified 126 patients undergoing eligible procedures. Figure 1 shows the population flowchart.

Of these 126 patients, 33 could not be offered the app for logistic reasons (either consultation location lacked documentation related to the app or patient being seen by a surgeon not involved in the pilot). Of the 93 patients approached, 36 were excluded because they had no smartphone, no email address or did not speak French. Of the remaining 57 patients, seven refused the connected follow-up because they felt overloaded by the context of their disease $(4 / 7)$ or the complexity of the system $(3 / 7)$. Thus, the willingness to accept mHealth was $88 \%$ for smartphone-equipped patients $(50 / 57)$. Characteristics of patients excluded or refusing the mHealth app are provided in Appendix S1.

Among the 50 patients included in the connected follow-up, two dropped out because of connection issues, one due to transfer to a rehabilitation centre and one due to a long hospital stay running over the study period. Three more patients dropped out because they deleted their e-profile, one due to the complexity and the other two because they felt no need. Table 1 summarizes the characteristics of the potentially eligible population $(n=126)$, approached population $(n=93)$ and population included in the final analysis $(n=43)$.

Patients with a connected follow-up $(n=43)$ had a median postoperative hospital stay of 4 days (range 2 20 days). Compliance with the ERAS pathway was $80 \%$ in the connected group. The connected patients completed all seven questionnaires in $72 \%(31 / 43)$ of cases. Among the remaining patients, two completed only the first five questionnaires, one patient completed the first two questionnaires and one completed only the first questionnaire. In addition, eight patients consulted their available information but did not answer any questionnaires. Figure 2 summarizes the distribution of patients' overall compliance with the connected follow-up period.

Figure I Population flowchart.

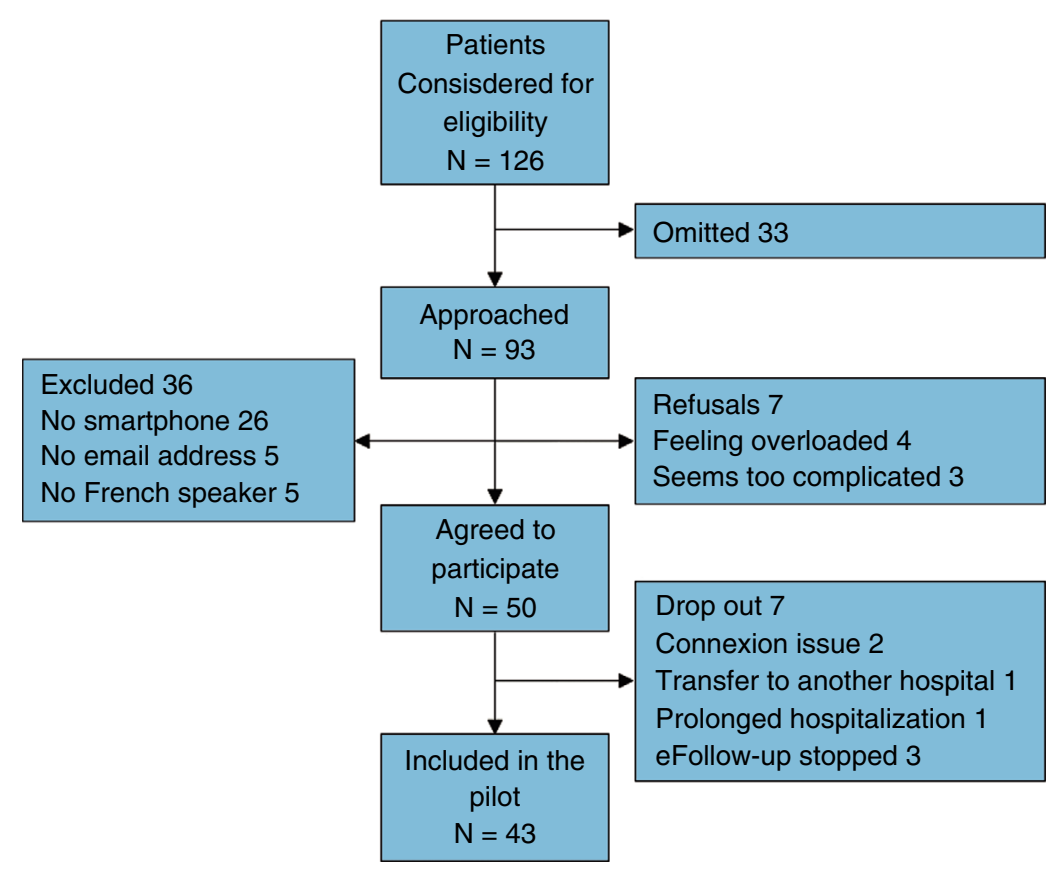


Table I Characteristics of the potentially eligible population $(n=126)$, approached population $(n=93)$ and the population with a connected follow-up $(n=43)$.

\begin{tabular}{|c|c|c|c|}
\hline Characteristic & $\begin{array}{l}\text { Potentially eligible population } \\
n=126\end{array}$ & $\begin{array}{l}\text { Approached population } \\
n=93\end{array}$ & $\begin{array}{l}\text { Population with a } \\
\text { connected follow-up } \\
n=43\end{array}$ \\
\hline Age (years), median (range) & $64.5(18-89)$ & $63(18-89)$ & $54(18-81)$ \\
\hline \multicolumn{4}{|l|}{ Gender, $n(\%)$} \\
\hline M & $73(58)$ & $52(56)$ & $26(60)$ \\
\hline $\mathrm{F}$ & $53(42)$ & $41(44)$ & $17(40)$ \\
\hline \multicolumn{4}{|l|}{ Interventions, $n(\%)$} \\
\hline Right colectomy & $32(25)$ & $21(23)$ & $10(23)$ \\
\hline Left colectomy & $24(19)$ & $18(19)$ & $10(23)$ \\
\hline Low anterior resection & $20(16)$ & $15(16)$ & $5(12)$ \\
\hline Sigmoidectomy & $18(14)$ & $13(14)$ & $5(12)$ \\
\hline Transverse colectomy & $8(6)$ & $5(5)$ & $2(5)$ \\
\hline Ileostomy closure & $7(6)$ & $6(7)$ & $4(9)$ \\
\hline Total colectomy & $9(7)$ & $7(8)$ & $4(9)$ \\
\hline Proctocolectomy & $2(2)$ & $2(2)$ & $2(5)$ \\
\hline Colostomy closure & $6(5)$ & $6(6)$ & $1(2)$ \\
\hline \multicolumn{4}{|l|}{ Disease, $n(\%)$} \\
\hline Cancer & $78(62)$ & $59(63)$ & $30(70)$ \\
\hline Non cancer & $48(38)$ & $34(37)$ & $13(30)$ \\
\hline \multicolumn{4}{|l|}{ Protocol followed, $n$ (\%) } \\
\hline Colorectal resection & - & - & $31(72)$ \\
\hline New stoma formation & - & - & $7(16)$ \\
\hline Stoma closure & - & - & $5(12)$ \\
\hline
\end{tabular}

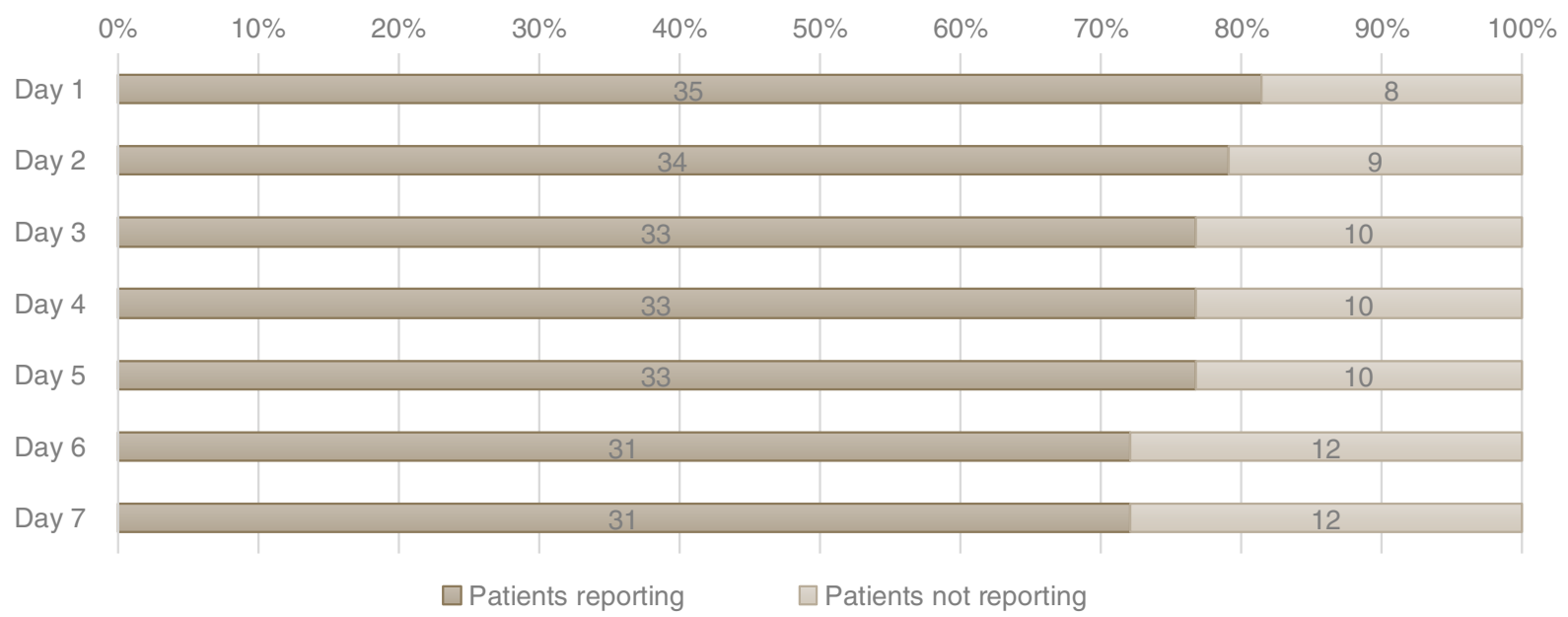

Figure 2 Distribution of patients' overall compliance to the questionnaires during the e-follow-up $(n=43)$.

The mHealth app displayed 12 AEs in 11 patients. No other AE was detected by clinical follow-up at the 30-day post-surgical visit. Table 2 summarizes AEs, detection patterns and resolution.

Healthcare providers responded to patient-generated alerts after a median time of $90 \mathrm{~min}$ (range 9$448 \mathrm{~min}$ ). A serious complication (anastomotic leak) was identified 3 days post-discharge in a patient who returned home 3 days after a laparoscopic low anterior resection with protective ileostomy. The patient was contacted by the PA and by the surgeon less than $5 \mathrm{~h}$ after the alert. The patient reported new cloudy liquid coming from the anus with light pain $(2 / 10)$ but no fever. He received an immediate appointment and a 
Table 2 Adverse events detected and managed through the mHealth app.

\begin{tabular}{|c|c|c|c|c|c|c|c|}
\hline $\begin{array}{l}\text { Type of adverse event } \\
N=12 \text { (11/43 patients) }\end{array}$ & $\begin{array}{l}\text { Detected } \\
\text { by the app }\end{array}$ & $\begin{array}{l}\text { Delay } \\
\text { post- } \\
\text { discharge } \\
\text { (day) }\end{array}$ & $\begin{array}{l}\text { Resolved } \\
\text { without } \\
\text { appointment }\end{array}$ & $\begin{array}{l}\text { Responder } \\
\mathrm{PA} / \mathrm{P}\end{array}$ & $\begin{array}{l}\text { Comm. } \\
\text { channel }\end{array}$ & $\begin{array}{l}\text { Reaction } \\
\text { time }\end{array}$ & Action realized \\
\hline Anastomotic leak & Yes & 3 & No & $\mathrm{PA} / \mathrm{P}$ & $\mathrm{TM}+\mathrm{PC}$ & $4 \mathrm{~h} 59 \mathrm{~min}$ & $\begin{array}{l}\text { Appointment, } \\
\text { rectoscopy + local } \\
\text { washing }\end{array}$ \\
\hline Urinary retention & Yes & 2 & No & PA & $\mathrm{TM}+\mathrm{PC}$ & $42 \mathrm{~min}$ & $\begin{array}{l}\text { Urgent appointment, } \\
\text { stop opioid }\end{array}$ \\
\hline Pain $\geq 6 / 10$ & Yes & 5 & Yes & $\mathrm{PA} / \mathrm{P}$ & $\mathrm{TM}$ & $2 \mathrm{~h} 18 \mathrm{~min}$ & $\begin{array}{l}\text { New prescription + } \\
\text { reassurance }\end{array}$ \\
\hline Pain $\geq 6 / 10$ & Yes & 5 & Yes & PA & $\mathrm{TM}$ & $34 \min$ & Reassurance + AA \\
\hline Pain $\geq 6 / 10$ & Yes & 5 & Yes & PA & $\mathrm{TM}$ & $11 \mathrm{~min}$ & Reassurance + AA \\
\hline Wound oozing (SP) & Yes & 1 & Yes & PA & $\mathrm{TM}$ & $46 \mathrm{~min}$ & $\begin{array}{l}\text { Wound dressing + } \\
\text { reassurance }\end{array}$ \\
\hline Wound anxiety & Yes & 2 & Yes & PA & TM & $4 \mathrm{~h} 51 \mathrm{~min}$ & $\begin{array}{l}\text { Reassurance } \\
\text { (photo visualized) }\end{array}$ \\
\hline Wound + MC anxiety & Yes & 3 & Yes & PA & $\mathrm{TM}$ & $2 \mathrm{~h} 49 \mathrm{~min}$ & $\begin{array}{l}\text { Reassurance } \\
\text { (photo visualized) }\end{array}$ \\
\hline Wound haematoma & Yes & 6 & Yes & PA & $\mathrm{TM}+\mathrm{PC}$ & $9 \mathrm{~min}$ & Reassurance \\
\hline Breathlessness (SP) & Yes & 4 & Yes & $\mathrm{PA}$ & $\mathrm{TM}$ & $14 \min$ & Reassurance \\
\hline Stoma bag leaking & Yes & 1 & Yes & PA & TM & $2 \mathrm{~h} 37 \mathrm{~min}$ & $\begin{array}{l}\text { Coordination with } \\
\text { stomatherapist }\end{array}$ \\
\hline Stoma bag leaking & Yes & 7 & Yes & PA & $\mathrm{TM}+\mathrm{PC}$ & $7 \mathrm{~h} 28 \mathrm{~min}$ & $\begin{array}{l}\text { Coordination with } \\
\text { stomatherapist }\end{array}$ \\
\hline
\end{tabular}

AA, analgesic advice; Comm. channel, communication channel; MC, menstrual cycle; P, physician; PA, physician assistant (clinical nurse); PC, phone call; SP, same patient; TM, text message.

rectoscopy showed a small $(5 \mathrm{~mm})$ anastomotic leak, with no need for further treatment.

The final satisfaction questionnaire regarding connected follow-up was completed by $30 \%$ of the patients $(13 / 43)$. The median age of responders was 50 years (range 34-61 years). The mean global satisfaction was $4 / 5$ (satisfied, SD =0.97). Finally, 12/13 patients said that they would use the connected follow-up again (one did not answer).

\section{Discussion}

\section{Principal findings}

The present pilot study suggests that follow-up with an mHealth app was accepted among smartphoneequipped colorectal patients, with high satisfaction reported by patients who responded to the questionnaire. Of note, all post-discharge complications were detected by the app and most could be managed in a timely manner without readmission or emergency consultation. A limitation, however, was the need for appropriate infrastructure and a third of patients could not be included due to the lack of necessary tools (smart phone, email address) and language proficiency.

To enable the widespread implementation of information technology such as mHealth apps in the medical field requires the need for better communication and information accessibility [21-23]. Semple et al. [17] reported the feasibility of mobile apps to monitor the postoperative quality of recovery at home after ambulatory surgery. In the present study, a similar feasibility was demonstrated for patients after major colorectal procedures. It is interesting to emphasize that older adults were inclined to use a connected strategy for their follow-up. There was no refusal among patients aged $>65$ years provided they had a smartphone and an e-mail account.

\section{Strengths and weaknesses}

The caregiver's median reaction time of 90 min was considered adequate for the AEs treated. The response delay included the time spent by the PA coordinating the next steps. In the case of the anastomotic leak, the patient received a response $5 \mathrm{~h}$ after the $\mathrm{AE}$ was 
discovered. During this time, the PA reviewed the patient's medical chart, excluded any life-threatening conditions and organized a rectoscopy with the surgeon in charge. The active search for complications can reduce the time for $\mathrm{AE}$ discovery but also avoid potential time lost in an emergency waiting room. Moreover, the structured communication via the platform and the easy availability of guidelines, framing the caregiver's response, facilitated the communication.

Although this pilot study was performed without additional staff, a large-scale implementation would probably require more staff, possibly further reducing the delay between alert and response.

The retrospective screening identified 126 patients undergoing eligible procedures, but only 93 of them were approached. This was because some patients were seen in a location lacking documentation presenting the concept and the functionalities of the mHealth app or were visited by a surgeon not involved in the pilot. Indeed, only the two colorectal surgeons involved in the pilot and the app administrator were authorized to recruit and enter patients in the app.

Among the overall number of approached patients ( $n=93), 62 \%$ had a smartphone. Although the present study shows various rates of smartphone based on age (97\% between 18 and 55 years old, $42 \%$ for patients $>55$ and $31 \%>65$ ), these levels are higher than reported in the literature [24]. It is important to emphasize that alternative follow-up solutions have to be made available for patients not equipped with smartphones or who cannot, or do not want to, use new technologies to communicate their medical data [25-27]. Nevertheless, the performance of the current connected solutions, as well as the users' personal experience and equipment, may be adequate to widely propose the mHealth app, which is a modern and practical way to improve care and information, to enhance quality and manage health costs.

This flexible communication channel demonstrated an $\mathrm{AE}$ in $26 \%$ of patients. Martinez et al. [28], in a cohort of 96 patients followed with a mobile-phonebased system, reported 31\% local wound problems. About $55 \%$ of these patients would have attended the ambulatory surgical unit if they had had no telemedicine. In the present study, 8/12 AEs (67\%) were solved with the use of personal text messages only and $4 / 12$ (33\%) with a text message and a phone call, potentially replacing time consuming and costly consultation. Only two patients needed a medical visit, which was organized via the platform. Moreover, all (12/12) AEs were managed in a short time by a PA $(10 / 12)$, with a physician required in two cases only.

SSI and pain are common concerns in modern medicine [29-33]. In the present study, $75 \%$ of AEs reported through the connected follow-up were either SSI or pain problems.

Improving access to caregivers via web platforms and apps allows them to reassure patients [34]. In our case, reassurance was possible through the app in two-thirds of AEs. Previous randomized controlled trials showed that smartphone-based pain assessment and real time caregivers' advice could result in fewer negative experiences $[34,35]$. The present study also confirms the value of mHealth apps to assess pain status. Mobilephone-based follow-up systems provide a method for comprehensive data acquisition and better $\mathrm{AE}$ reporting [36]. Overall, this new communication tool shows how early hospital discharge can be a challenge for patients and how caregivers must be aware of and offer solutions to the challenges faced.

The difference of 10 years (64.5 vs 54) between the median age of approached patients and followed patients is due to the exclusion criteria, the main reason being that older adults were less equipped with a smartphone and/or email address. However, the four cases where patients refused the connected solution for motives other than the complexity of the tool involved young, equipped patients $(18,19,43$ and 44 years old). These patients felt overwhelmed by their disease and its treatment, with medical appointments and planned hospitalizations, thus refusing for psychological reasons. Four patients found the solution too complicated and refused participation $(3 / 4)$ or stopped their electronic follow-up $(1 / 4)$. It is necessary to learn from previous deployments in order to provide an optimal solution to the largest possible population in the future [24].

All patients with connected follow-up received standard ERAS information on their e-profiles, while nonconnected patients received the same information in printed form. Compliance with the ERAS objectives was higher among the connected group (80\%) than the non-connected group (69.5\%). This compliance difference is probably biased by the selection criteria of the connected group (new technology users and French speakers), but it is possible that rapid access to ERAS information directly on the smartphone contributed to this result [21].

\section{Future research}

Because the main reasons for exclusion were the absence of connection tools and the language barrier, it is important to overcome these issues in the future. The translation of the app's content into locally frequently spoken languages is a prerequisite for the next implementation. A major implication of patients' relatives, 
partnerships with home nursing services and the provision of a stock of smartphones to lend to patients are all concepts to be developed for the recruitment of patients who do not use smartphones and to improve the engagement of users.

Outpatient surgery also contributes to shifting the onset of AEs from the hospital to the patient's home. For this reason, a future implementation will also include outpatient procedures (proctological surgery).

As more resources will be invested in the next implementation, it is planned to prospectively record satisfaction data.

Finally, although investment in the app seemed reasonable, larger studies will permit an assessment of the cost-benefit of the mHealth app.

\section{Limitations}

The limitations of this study were inherent to its retrospective character. The small sample size due to the exclusion of potential users was also a limitation. Moreover, while not considered in the feasibility assessment, missed cases were also high. This was mostly due to a ramp-up period during the first months of implementation. Furthermore, data regarding the socioeconomic and sociocultural status of the 93 approached patients have not been collected.

Our response rate to the satisfaction questionnaire was low $(30 \%)$, although it reflects the average response rate to online surveys $(33 \%)$ and the even lower in-app survey response rate $(13 \%)$ [37]. This could be due to survey fatigue, a lack of incentive or to a progressive disengagement as patients progressively recover from surgery.

The willingness of the 36 excluded patients to engage in mHealth was not reported, although there was probably a 'theoretical' acceptance rate among them too. It was decided to estimate the willingness using only candidates who had the choice to use the app or not $(n=57)$.

Finally, there was no information about the use of other communication channels outside the defined office hours.

\section{Conclusion}

Communication between caregivers and patients needs to be improved [38] and telemedicine may be a solution. Today, mHealth apps seem to provide a tool for early detection of post-discharge complications, offering information delivery and patient empowerment.

This study suggests that follow-up with an mHealth app is feasible but raises the issue of the broad inclusion of patients. This may be overcome in the future with proper organization and improved patient information in order to increase their motivation. Additional resources will also be needed to provide $24 / 7$ surveillance. These efforts, however, appear justified to improve patients' safety and satisfaction and to avoid costly and unnecessary readmissions or consultations.

\section{Acknowledgements}

The authors thank the clinical nurses Pauline Valles and Seada Cirak, the ERAS coordinator Valérie Addor, Alain Junger (chief nurse informatics officer) as well as the legal affairs Department of the CHUV for their important contribution during the mHealth app implementation period. This study received no specific grant from any funding agency.

\section{Conflict of interests}

The authors have no competing interests to declare.

\section{References}

1 Connors HR. Telecommunications and rural health care. Adv Nurse Pract 1998; 6: 40-4, 66.

2 Dolan B. (2010). Timeline: The iPhone as a medical tool. Retrieved from https://www.mobihealthnews.com/ 2580/timeline-the-iphone-as-medical-tool (accessed 7 November 2018).

3 WHO. mHealth New Horizons for Health Through Mobile Technologies. Volume 3. Global Observatory for eHealth Series, 2011. Geneva, Switzerland: WHO, World Health Organization.

4 Aitken M, Lyle J. Patient Adoption of mHealth. Report by the IMS Institute for Healthcare Informatics, 2015. Retrieved from https://www.iqvia.com/-/media/iqvia/ pdfs/institute-reports/patient-adoption-of-mhealth.pdf (accessed 12 November 2018).

5 De Jongh T, Gurol-Urganci I, Vodopivec-Jamsek V. Mobile phone messaging for facilitating self-management of long-term illnesses. Cochrane Database Syt Rev 2012; 12: CD007459.

6 Martin D, Hübner M, Moulin E et al. Timing, diagnosis, and treatment of surgical site infections after colonic surgery: prospective surveillance of 1263 patients. J Hosp Inf 2018; 100: 393-9.

7 Agri F, Griesser AC, Lécureux E et al. Assessment of avoidable readmissions in a visceral surgery department with an algorithm: methodology, analysis and measures for improvement. World J Srug 2019; 43: 107-16.

8 Merkow RP, Ju MH, Chung JW. Underlying reasons associated with hospital readmission following surgery in the United States. JAMA 2015; 313: 483-95.

9 Royal College of Surgeons of England, Department of Health. Emergency Surgery: Standards for Unscheduled Care, 2011.London: RCS/DH.

10 National Confidential Enquiry into Patient Outcome and Death. An age old problem: a review of the care received 
by elderly patients undergoing surgery. London: NCEPOD, 2010.

11 Jestin P, Nilsson J, Heurgren $M$ et al. Emergency surgery for colonic cancer in a defined patient population. $\mathrm{Br} J$ Surg 2005; 92: 94-100.

12 Organisation for Economic Cooperation and Development. Health at a Glance 2017: OECD Indicators, 2017. Paris: OECD.

13 Busse R, Geissler A, Aaviksoo A et al. Diagnosis related groups in Europe: moving towards transparency, efficiency, and quality in hospitals? BMJ 2013; 346: f3197.

14 Gignoux B, Pasquer A, Vulliez A et al. Outpatient colectomy within an enhanced recovery program. J Visc Surg 2015; 152: 11-5.

15 Gignoux B, Gosgnach M, Lanz T et al. Short-term outcomes of ambulatory colectomy for 157 consecutive patients. Ann Surg 2019; 270: 317-321. https://doi. org/10.1097/SLA.0000000000002800

16 Martinez-Ramos C, Cerdàn MT, López RS. Mobile phone-based telemedicine system for the home follow-up of patients undergoing ambulatory surgery. Telemed $J E$ Health 2009; 15: 531-7.

17 Semple JL, Sharpe S, Murnaghan ML et al. Using a mobile app for monitoring post-operative quality of recovery of patients at home: a feasibility study. JMIR Mhealth Uhealth 2015; 3: el8.

18 Jaensson M, Dahlberg K, Eriksson M et al. The development of the recovery assessment by phone points (RAPP): a mobile phone app for postoperative recovery monitoring and assessment. JMIR Mhealth Uhealth 2015; 3: e86.

19 Hwa K, Wren SM. Telehealth follow-up in lieu of postoperative clinic visit for ambulatory surgery: results of a pilot program. JAMA Surg 2013; 148: 823-7.

20 Vella MA, Kummerov Broman K, Tarpley JL et al. Postoperative telehealth visits: assessment of quality and preference of veterans. JAMA Surg 2015; 150: 1197-9.

21 Ventola CL. Mobile devices and apps for health care professionals: uses and benefits. P T 2014; 39: 356-64.

22 Mosa AS, Yoo I, Sheets L. A systematic review of health care apps for smartphones. BMC Med Inform Dec Mak 2012; 12: 67.

23 Moodley A, Mangino J, Goff D. Review of infectious disease applications for iPhone/iPad and Android: from pocket to patient. Clin Infect Dis 2013; 57: 1145-54.

24 Wiseman JT, Fernandes-Taylor S, Barnes ML. Conceptualizing smartphone use in outpatient wound assessment: patients' and caregivers' willingness to use technology. $J$ Surg Res 2015; 198: 245-51.

25 Atienza AA, Zarcadoolas C, Vaughon W. Consumer attitudes and perceptions on $\mathrm{mHealth}$ privacy and security: finding from a mixed-methods study. J Health Commun 2015; 20: 673-9.

26 Serrano KJ, Yu M, Riley WT. Willingness to exchange health information via mobile devices: finding from a population-based survey. Ann Fam Med 2016; 14: 34-40.
27 McMahon SK, Lewis B, Oakes M. Older adults' experiences using a commercially available monitor to self-track their physical activity. JMIR Mhealth Uhealth 2016; 4: e35.

28 Martinez-Ramos C, Cerdàn MT, López RS. Mobile phone-based telemedicine system for the home follow-up of patients undergoing ambulatory surgery. Telemed $e^{-}$ Health 2009; 15: 531-9.

29 Mangram AJ, Horan TC, Pearson ML. Guideline for prevention of surgical site infection. Am J Infect Control 1999; 27: 97.

30 Whitby M, McLaws ML, Collpoy B. Post-discharge surveillance: can patients reliably diagnose surgical wound infections? J Hosp Infect 2002; 52: 155.

31 Gustafsson UO, Scott MJ, Schwenk W. Guidelines for perioperative care in elective colonic surgery: Enhanced Recovery After Surgery (ERAS $\left.{ }^{\circ}\right)$ Society recommendations. World J Surg 2013; 37: 259-84.

32 Nygren J, Thacker J, Carli F. Guidelines for perioperative care in elective rectal/pelvic surgery: Enhanced Recovery After Surgery (ERAS $\left.{ }^{\circledR}\right)$ Society recommendations. World $J$ Surg 2013; 37: 285-305.

33 Ljungqvist $\mathrm{O}$, Scott $\mathrm{M}$, Fearon KC. Enhanced recovery after surgery: a review. JAMA Surg 2017; 152: 292-8.

34 Kristjánsdóttir OB, Fors EA, Eide E. A smartphone-based intervention with diaries and therapist-feedback to reduce catastrophizing and increase functioning in women with chronic widespread pain: randomized controlled trial. $J$ Med Intern Res 2013; 15: e5.

35 Boulos MN, Brewer AC, Karimkhani C. Mobile medical and health apps: state of the art, concerns, regulatory control and certification. J Public Health Inform 2014; 5: 229.

36 Stomberg MW, Platon B, Widé n A. Health information: what can mobile phone assessment add? Perspect Health Inf Manag 2012; 9: 1-10.

37 Lindemann N. What's the Average Survey Response Rate? [2019 benchmark]. Available at https://surveyanyplace.c om/average-survey-response-rate/ (accessed 1 November 2019).

38 Admina M, Kehlet H, Tomlinson GA. Enhanced recovery pathways optimize health outcomes and resources utilization: a meta-analysis of randomized controlled trials in colorectal surgery. Surgery 2011; 149: 830-40.

\section{Supporting Information}

Additional Supporting Information may be found in the online version of this article:

Appendix S1. Characteristics of patients excluded $(n=36)$ or refusing $(n=7)$ the mHealth app. 